Communications in Physics, Vol.23, No. 2 (2013), pp. 171-177

\title{
INFLUENCE OF THE FREQUENCY-CHIRP ON PULSE IN PASSIVELY MODE-LOCKING OPTICAL FIBER RING LASER
}

\author{
BUI XUAN KIEN \\ Electric Power University \\ 235 Hoang Quoc Viet Str, Tu Liem Dist., Hanoi, Vietnam \\ Email: kiendhv2000@yahoo.com \\ TRAN HAI HUNG \\ Nghe An Pedagogical College \\ TRINH DINH CHIEN \\ Hanoi University of Science, \\ Vietnam National University, Hanoi \\ 334 Nguyen Trai Str., Thanh Xuan Dist., Hanoi, Vietnam \\ Received 05 March 2013; revised manuscript received 31 March 2013
}

\begin{abstract}
We consider a model of a passively mode-locking fiber ring laser bult using a saturable absorber and a chirped fiber Bragg grating to balance dispersion and nonlinearity. The evolution of the slowly envelope of the optical field in a loop fiber subject to dispersion, Kerr nonlinearity, frequency- chirp and nonlinear absorption is given by the generalized complex Ginzburg-Landau equation. The influence of the frequency-chirp on the pulse is simulated and discussed, and the stationary conditions concerning the chirp parameter are found out for our laser.
\end{abstract}

\section{INTRODUCTION}

In recent years there has been an extensive growth in research activities focused on developing ultrashort $(<10 \mathrm{ps})$ pulse sources based on rare-earth-doped-fiber [1-4]. The short pulse fiber-lasers have been passively mode-locked by using the chirp-fiberBragg-grating (C-FBG) to control dispersion, the polarization controller, the semiconductor saturable absorber mirror (SESAM) or the multiple quantum well saturable absorber (MQW-AS) [5]. The theory of mode-locking is based on the master equation derived under the condition that nonlinear changes to the intra-cavity pulse must be small per round-trip and fast saturable absorber action [6]. The carrier dynamics is adiabatically eliminated due to the sub-picosecond material response time and pulse widths of order ten picosecond. To obtain analytic results, the form of a complex Ginzburg-Landau equation is used, where the nonlinear contributions of the saturable absorber are approximated by an expansion of the field amplitude to fifth order [7]. The simulated results in previous works [5,7], are focused on the generation solitonic shape of the output pulse only, so the initial pulse envelope has been chosen by using the hyperbolic secant solution with no chirp. 
In this paper, we present numerical studies of a ring fiber laser passively modelocked by MQW saturable absorber with chirped fiber Bragg grating. The simulation is used for the initial Gaussian pulse with frequency- chirp.

\section{MODELING AND SIMULATION}

Our model of passively mode-locked ring fiber laser is illustrated in Fig. 1.

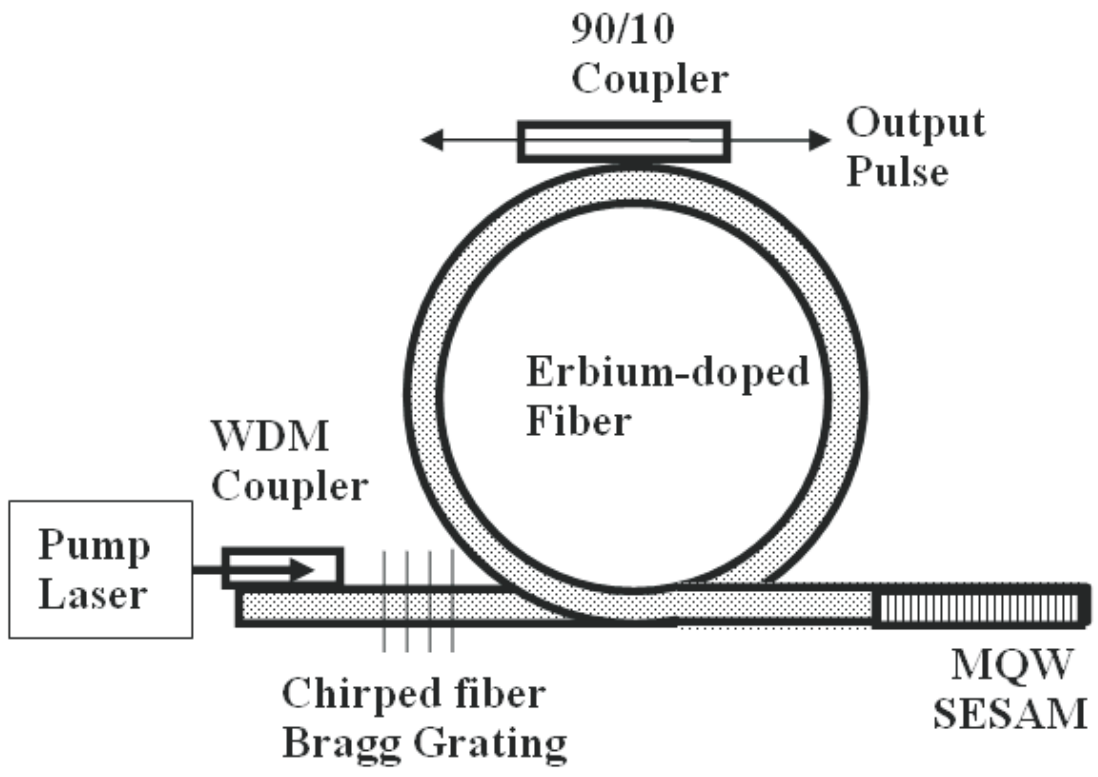

Fig. 1. Schematic of the passively mode-locked ring fiber laser.

The theory of mode-locking is based on the complex Ginzburg-Landau equation derived under the condition that round-trip changes to the intra-cavity pulse are small. The saturable absorption was modeled by two-level system rate equation, which accounts for the free carrier that generated refractive index changes in the semiconductor absorber. The cavity dispersion is determined from the chirped fiber grating and the cavity losses are estimated basing on the properties of the MQW sample.

The average equation belonging to the class of generated complex Ginzburg-Landau equations has a form

$$
\frac{\partial A}{\partial z}=i\left(D \frac{\partial^{2}}{\partial t^{2}}+\delta_{3}|A|^{2}\right) A+\left(D_{g} \frac{\partial^{2}}{\partial t^{2}}+g-l+\gamma_{3}|A|^{2}-\gamma_{5}|A|^{4}\right) A
$$

Here $A$ is the optical field complex amplitude, scaled so that its square has units of optical power; $t$ is the time variable and $z$ is the distance divided by the cavity round-trip length $L_{c}$. The first two terms on the right hand side are imaginary contributions; $D$ is the cavity dispersion, $\delta_{3}$ is related to the effective Kerr nonlinearity of the cavity. The last four terms have real coefficients with the following physical meaning: $D_{g}$ is the gain and intracavity 
filter bandwidth limits; $l-g$ is the net loss, where $l$ and $g$ are linear losses and gain per round-trip, respectively. Other physical parameters are defined as

$$
D=-\frac{\beta_{2} L_{c}+D_{g r}}{2}, D_{g}=\frac{g}{\Omega_{g}^{2}}+\frac{1}{\Omega_{f}^{2}}, \delta_{3}=\gamma L_{c}+\alpha \gamma_{3}
$$

where $\beta_{2}$ is the group velocity dispersion (GVD) for the fiber component, $L_{c}$ is the optical cavity round-trip length, $D_{g r}$ is the dispersion from the chirped grating. Further parameters are: $\Omega_{g(f)}$, gain (filter) bandwidth, the nonlinear absorption parameters are given by $\gamma_{3}=q_{0} / P_{s}$ and $\gamma_{5}=q_{0} / P_{s}^{2}$, where $q_{0}$ is the measured linear loss and $P_{s}$ is the saturation power of the MQW element. The nonlinear dispersion has two contributions, one from the fiber $\gamma L_{c}$, where $\gamma=2.6 / W k m$ is the fiber's Kerr coefficient [5,7], and the other from the saturable absorber. The enhancement parameter, $\alpha$, is adjusted to include the nonlinear index change of the saturable absorber.

The simulations applied the beam propagating algorithm. The initial pulse envelope will be chosen by the frequency -chirp Gaussian profile solution. The initial pulse is

$$
A=A_{0} \exp \left[-\frac{(1+i C) t^{2}}{2 T_{0}^{2}}\right]
$$

Using (3) to (1), after some arrangements we have

$$
\begin{aligned}
\frac{\partial A}{\partial z}= & i\left(\frac{D}{T_{0}^{2}}+\delta_{3}\left|A_{0}\right|^{2} e^{-\frac{t^{2}}{T_{0}^{2}}}+\frac{C D_{g}}{T_{0}^{2}}\right) A \\
& +\left(-\frac{D C}{T_{0}^{2}}+\frac{D_{g}}{T_{0}^{2}}+\frac{2 D_{g}\left(1-C^{2}\right) t^{2}}{T_{0}^{4}}+g-l+\gamma_{3}\left|A_{0}\right|^{2} e^{-\frac{t^{2}}{T_{0}^{2}}}-\gamma_{5}\left|A_{0}\right|^{4} e^{-\frac{2 t^{2}}{T_{0}^{2}}}\right) A
\end{aligned}
$$

The physical parameters for our cavity are: $L_{c}=15 \mathrm{~m}, D_{g r}=-13 \mathrm{ps}^{2}, \beta_{2}=-15$ $\mathrm{ps}^{2} / \mathrm{km}, D_{g}=0.015 \mathrm{ps}^{2}, l=0.2 / \mathrm{m}, g=0.5 / \mathrm{m}$. The parameters $q_{0}=0.55$ and $P_{s}=$ $5 \mathrm{~mW}$, give $\gamma_{3}=0.11 / \mathrm{mW}$, and $\gamma_{5}=0.5 / \mathrm{mW}^{2}$. The choice $\alpha=5$, yields $\delta_{3}=0.5 / \mathrm{mW}$.

There are three open parameters in our model, the gain $g$, the enhancement parameter $\alpha$, and the chirp parameter $C$. The two first parameters have been discussed and presented in previous work [5]. In this work we focus only on the influence of the chirp parameter on the output pulse.

We consider the initial pulse appeared in the fiber is a frequency-chirp Gaussian with the peak amplitude of $A_{0}=1 \mathrm{~mW}$ and the half of duration of $T_{0}=10 \mathrm{ps}$. The input pulse and pulses after some round-trip are simulated using equation (4) and illustrated in Fig. 2 for case without frequency-chirp, $C=0$. Then it can see that the peak power of the pulses are amplified and its duration smoothly increased after each round-trip.

These simulated results are in good agreement with that given in work of Spaulding [8], in which the nonlinear dynamics of the mode-locking optical fiber ring laser are theoretically investigated. For the pulse with frequency-chirp of $C=-5$ and $C=-10$, the output pulses are illustrated in Fig. 3.

After each round-trip the peak power of the pulse is reduced, however, the reduction magnitude is about $1 \%$ if $\mathrm{C}=-10$. Meanwhile its duration is appreciably increased about two times $(\sim 20 \mathrm{ps})$ after $20^{\text {th }}$ round-trip, that means the pulse is broadened by the 


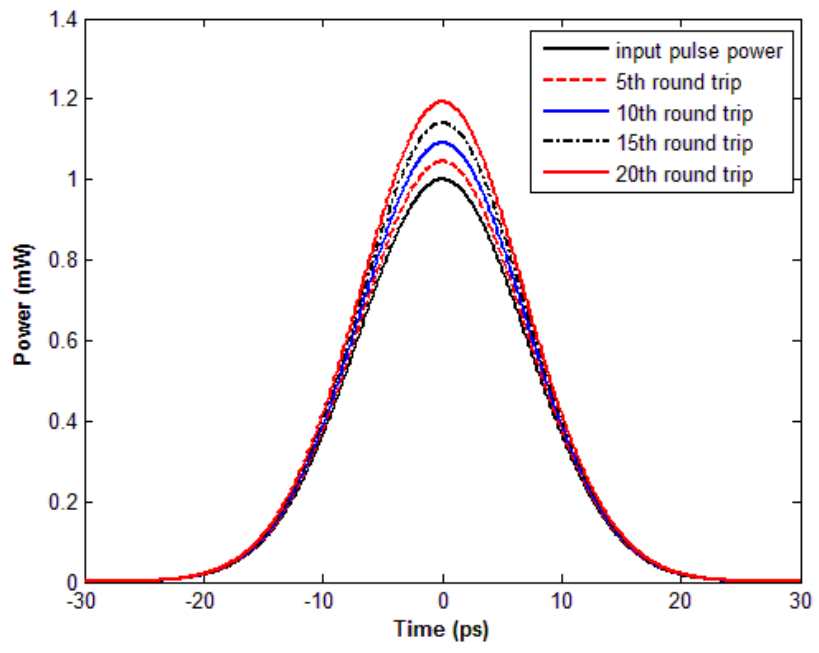

Fig. 2. Simulated without-chirp Gaussian pulses after round-trips in laser cavity

unnormalous-dispersion and down-chirp [9]. Moreover, the peak reduction and duration broadening depend on the chirp parameter, which is shown in Fig. 3, where reduction magnitude is about $1 \%$ and $0.4 \%$, and duration is about $20 \mathrm{ps}$ and $13 \mathrm{ps}$ after $20^{\text {th }}$ roundtrip, for $\mathrm{C}=-10$ and $\mathrm{C}=-5$, respectively. Conversely, for the case of $C=10$ (see Fig. 4) the peak power of the output pulse is appreciately amplified about two times after $20^{\text {th }}$ round-trip. But the its duration is quite no changed. In other ways it can say that the pulse with up-chirp should be compressed in the laser cavity.

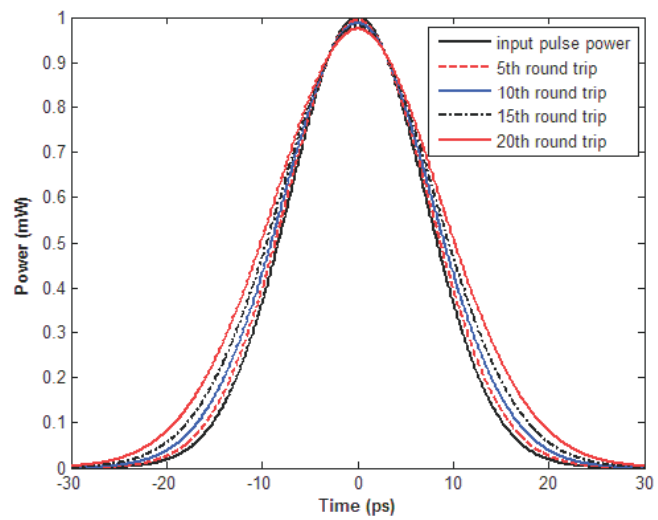

(a)

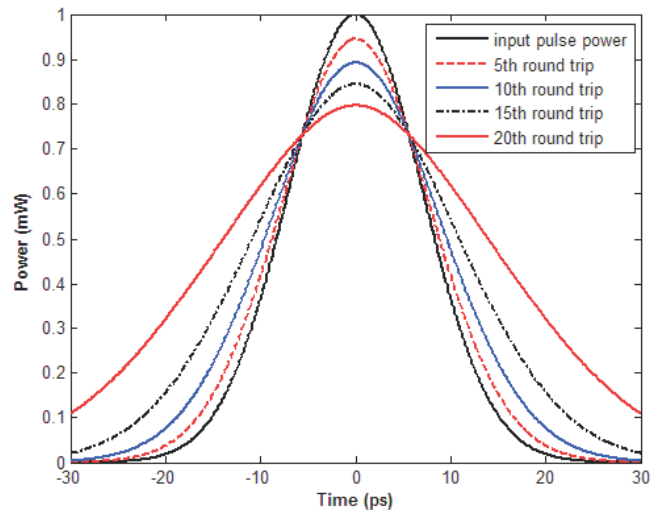

(b)

Fig. 3. Simulated down-chirp Gaussian pulses after round-trips in laser cavity. a) $C=-5$, b) $C=-10$. 


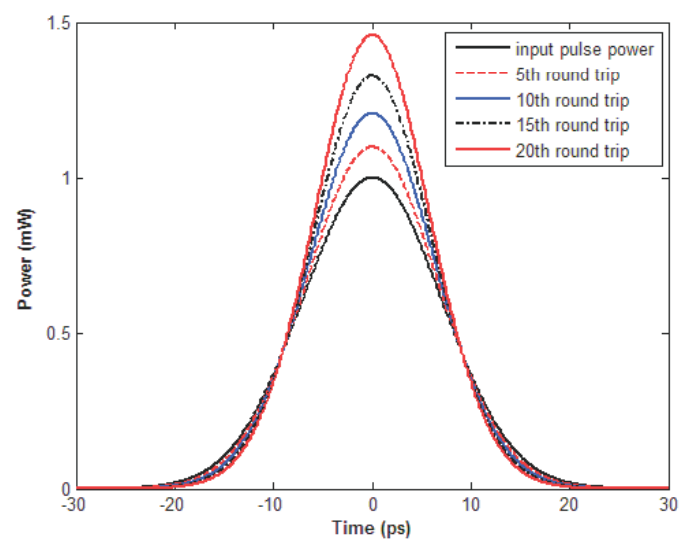

(a)

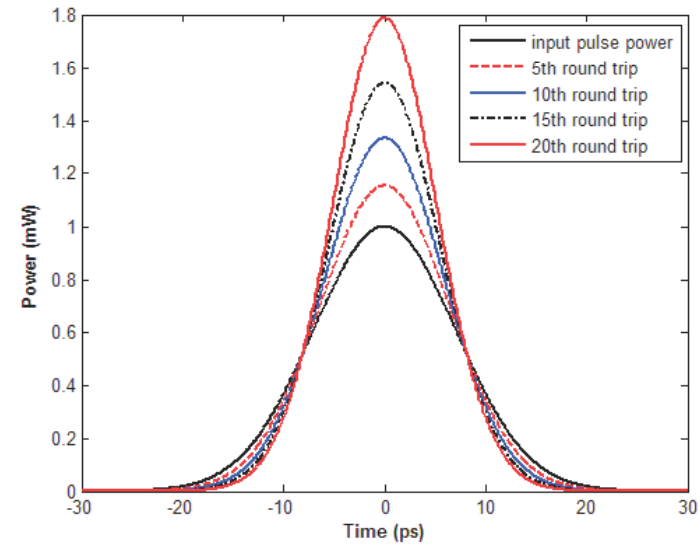

(b)

Fig. 4. Simulated up-chirp Gaussian pulses after round-trips in laser cavity. a) $C=5$, b) $C=10$.

The character points in figures 3 and 4 are discussed for two cases of the dispersion with two distinguish values of the frequency chirp, only. To confirm this character, the output pulses after $20^{t h}$ round-trip with some values of the frequency-chirp parameter for the case of anomalous dispersion are simulated and illustrated in Fig. 5. The results shows that if the amplifier fiber is the anomalous dispersive one, the output pulses will be more and more compressed when the frequency chirp parameter is positive and increases, but they will be broadened when the frequency chirp parameter is negative and decreases.

The results presented in Fig. 5 are similar to that in the normalous dispersion laser cavity with opposite sign of the frequency chirp parameter.

From above discussions, it is clear that the frequency chirp parameter plays an impotant role in pulsing process in the laser cavity with dispersion.

The amplification process in laser cavity will be continuous round-trip till the stationary condition, for which the pulse will not changed, will be satisfied. It means that the propagating length of the intracavity pulse must be chosen so that $\partial A / \partial z=0$. From (4), we can find out the condition for the initial pulse

$$
\left.\left|\frac{D}{T_{0}^{2}}+\delta_{3}\right| A_{0}\right|^{2} e^{-\frac{t^{2}}{T_{0}^{2}}}+\frac{C D_{g}}{T_{0}^{2}} \mid=0
$$

and $-g+l+\frac{D C}{T_{0}^{2}}-\frac{D_{g}}{T_{0}^{2}}-\frac{2 D_{g}\left(1-C^{2}\right) t^{2}}{T_{0}^{4}}-\gamma_{3}\left|A_{0}\right|^{2} e^{-\frac{t^{2}}{T_{0}^{2}}}+\gamma_{5}\left|A_{0}\right|^{4} e^{-\frac{2 t^{2}}{T_{0}^{2}}}=0$

We consider the stationary condition satisfies at every time, $t$, in the duration of the pulse i.e. satisfies at $t=0$. From given initial pulse (3) and condition (5), the condition for parameters of the initial pulse is given by

$$
T_{0}^{2}\left|A_{0}\right|^{2}<\frac{\left|D+C D_{g}\right|}{\delta_{3}}
$$




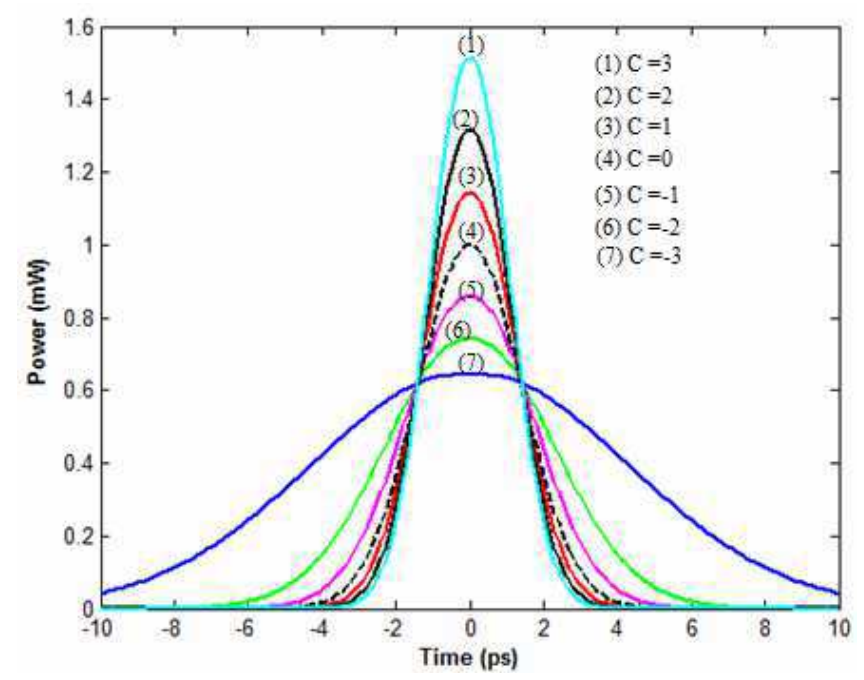

Fig. 5. Simulated up-chirp Gaussian pulses after $20^{\text {th }}$ round-trip in the anomalous dispersion laser cavity with some frequency chirp parameters.

and condition for the energy balance of a stationary solution is given by

$$
g-l=\frac{D C}{T_{0}^{2}}-\frac{D_{g}}{T_{0}^{2}}-\gamma_{3}\left|A_{0}\right|^{2}+\gamma_{5}\left|A_{0}\right|^{4}
$$

With the physical parameters given above and $\mathrm{C}=5$ the initial pulse with half duration of $T_{0}^{2}=10 \mathrm{ps}$, consequently, with peak power of $P_{0}=A_{0}^{2}=0.134 \mathrm{~mW}$. This initial pulse evolves in cavity using the Ginzburg-Landau equation until a new steady-state is achieved. If initial and final solutions are quite close, which demonstrates that the pulse is well approximated by soliton parameters. This problem will be presented in the future, in which the condition to crease the optical soliton should be interested.

\section{CONCLUSION}

The passively mode-locked ring Erbium-doped fiber laser is designed using the MQW saturable absorber and chirped fiber Brag grating. The evolution of the frequency-chirp Gaussian pulse in the cavity is given by the Ginzburg-Landau equation. The influence of the chirp parameter is simulated for the cases of the Gaussian pulse without-chirp, down-chirp and up-chirp. The shaping of the pulse, broadening, narrowing, amplifying and reduction, in the cavity depends on the the chirp parameter, $\mathrm{C}$. This shaping process of the pulse depends not on chirp parameter only, but also on other physical parameters, and stationary process to generate solitonic pulse will appear under the certain conditions, which will be investigated in the future.

\section{REFERENCES}

[1] M. E. Fermann, Appl. Phys. B58 (1994) 197-209.

[2] M. E. Fermann, A. Galvanauskas, G. Sucha and D. Harter, Appl. Phys. B 65 (1997) 259-275. 
[3] O. G. Akhotmikov, L. Gomes, N. Xiang and T. Jouhti, Optics Letters, 28, 17 (2003) 1522-1524.

[4] F. O. Ilday, J. hen, and F. X. Kartner, Optics Express, 13, 7 (2005) 2716-2721.

[5] J. W. Haus et al, Optics Communication 174 (2000) 205-214.

[6] F. X. Kartner et al, IEEE J. Quantum Electon. 2 (1996) 540.

[7] G. Shaulov, I. Gabitov, J. W. Haus, Optics communication 164 (1999) 39-45.

[8] K. M. Spaulding et al, Opt. Soc. Am. B 19, 5 (2002) 1045-1054.

[9] G. P. Agrawal, Nonlinear fiber optics, Fourth Edition. Academic press. (2007) 50-58. 